\title{
Geometry of the Stable Ruled Surface Over an Elliptic Curve
}

\author{
Arame Diaw ${ }^{1}$
}

Received: 28 February 2019 / Accepted: 17 July 2020 / Published online: 3 August 2020

(c) The Author(s) 2020

\section{Abstract}

We consider the stable ruled surface $S_{1}$ over an elliptic curve. There is a unique foliation on $S_{1}$ transverse to the fibration. The minimal self-intersection sections also define a 2-web. We prove that the 4 -web defined by the fibration, the foliation and the 2 -web is locally parallelizable.

Keywords Elliptic curve $\cdot$ Ruled surface $\cdot$ Riccati foliation and Singular web

\section{Contents}

1 Introduction . . . . . . . . . . . . . . . . . . . . . . . . 6 645

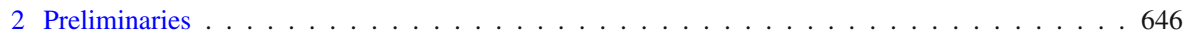

2.1 Some Properties on An Elliptic Curve . . . . . . . . . . . . . . . . . . . . . . . . . . 646

2.2 Ruled Surface Over An Elliptic Curve . . . . . . . . . . . . . . . . . . . . . . . . . . . . 647

3 Geometry of the Ruled Surface $S_{1} \ldots \ldots \ldots \ldots \ldots$. . . . . . . . . . . . . 650

3.1 Study of Special Leaves of the Riccati Foliation $\mathcal{R} \ldots \ldots$. . . . . . . . . . . . . . . . . 653

3.2 The Geometry of the 4 -Web W . . . . . . . . . . . . . . . . . . . . . . . . 654

4 Geometry of 4-Web W After Elementary Transformations . . . . . . . . . . . . . . . . . . 655

4.1 The Riccati Foliation on $S_{1}$ After Elementary Transformations . . . . . . . . . . . . . . . 657

4.2 The 2-Web After Elementary Transformations . . . . . . . . . . . . . . . . . . . . 658

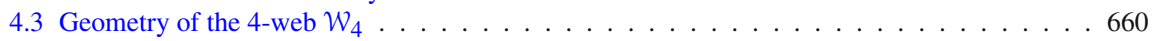

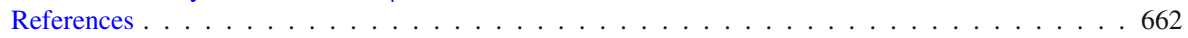

\section{Introduction}

Let $C$ be an elliptic curve on $\mathbb{C}$. In 1955, Atiyah proved in Atiyah (1955) that, up to isomorphism, there are only two indecomposable ruled surfaces over $C$ : the semistable ruled surface $S_{0} \rightarrow C$ and the stable ruled surface $S_{1} \rightarrow C$. In this article, we

A. Diaw: supported by ANR-16-CE40-0008 Project "Foliage".

$凶 \quad$ Arame Diaw

aramdiaw2@gmail.com

1 Univ Rennes, CNRS, IRMAR - UMR 6625, 35000 Rennes, France 
study the geometry of the stable ruled surface. In fact, the surface $S_{1}$ can be seen as the suspension over $C$ of the unique representation onto the dihedral group $<-z, \frac{1}{z}>$ (see Loray and Pérez 2009, p. 23). Thus, we have a Riccati foliation $\mathcal{R}$ on $S_{1}$ such that the generic leaf is a cover of degree 4 over $C$ and it is the unique foliation transverse to the fibration. On the other hand, the holomorphic section $\sigma: C \rightarrow S_{1}$ have selfintersection $\sigma . \sigma \geq 1$ and those having exactly $\sigma . \sigma=1$ form a singular holomorphic 2 -web $\mathcal{W}$. Finally, taking into account the fibration we have a singular holomorphic 4-web on $S_{1}$. The aim of this article is to study the geometry of this 4-web composed by the Riccati foliation, the 2-web $\mathcal{W}$ and the $\mathbb{P}^{1}$-fibration $\pi: S_{1} \rightarrow C$.

Our first result is the following:

Proposition 1.1 The discriminant $\Delta$ of the 2 -web $\mathcal{W}$ defined by the +1 self-intersection sections on $S_{1}$ is a leaf of the foliation $\mathcal{R}$.

Using the isomorphism between the curve $C$ and its jacobian, we have the main result

Theorem 1.2 There exists a double cover $\varphi: C \times C \rightarrow S_{1}$ ramified on $\Delta$ on which the lifted 4-web $\boldsymbol{W}$ is parallelizable.

Firstly, we show these results using only the properties of an elliptic curve and its jacobian and after we use the theory of birational geometry to illustrate our results with computations on a trivialization $S_{1} \rightarrow C \times \mathbb{P}^{1}$.

This paper is part of my thesis work under the direction of Frank Loray and Frédéric Touzet.

\section{Preliminaries}

\subsection{Some Properties on An Elliptic Curve}

Let $C=\left\{(x, y) \in \mathbb{C}^{2}, y^{2}=x(x-1)(x-t)\right\} \cup\left\{p_{\infty}\right\}$, where $t \in \mathbb{C} \backslash\{0,1\}$ be an elliptic curve. The set of points of $C$ forms an abelian group with $p_{\infty}$ as the 0 element and the points $p_{i}=(i, 0)$ where $i=0,1, t$, are the points of order 2 on $C$. Note that the map

$$
\left\{\begin{array}{l}
I: C \rightarrow C \\
(x, y) \mapsto(x,-y)
\end{array}\right.
$$

is an automorphism of $C$ which fixes the points of order 2: it is the standard involution of the curve $C$.

If we denote $\operatorname{Jac}(C)$, the jacobian of $C$, we have:

Lemma 2.1 There exists a group isomorphism between $C$ and its jacobian defined by this following map:

$$
\left\{\begin{array}{l}
C \longrightarrow J a c(C) \\
p \longmapsto[p]-\left[p_{\infty}\right]
\end{array}\right.
$$


From now on, we will use this isomorphism between the additive group structure of $\left(C, p_{\infty}\right)$ and the group structure of its jacobian.

\subsection{Ruled Surface Over An Elliptic Curve}

Let $C$ be a smooth curve on $\mathbb{C}$.

Definition 2.2 A ruled surface over $C$ is a holomorphic map of two dimensional complex variety $S$ onto $C \pi: S \rightarrow C$ which makes $S$ a $\mathbb{P}^{1}$-fibration over $C$.

Example 2.3 The fiber bundle associated to a vector bundle of rank 2 over $C$ is a ruled surface. We denote it $\mathbb{P}(E)$.

Conversely, we have the following theorem whose detailed proof is in Hartshorne (1977):

Theorem 2.4 Let $\pi: S \rightarrow C$ be a ruled surface over $C$ :

1. there exists a vector bundle $E$ of rank 2 over $C$ such that $S=\mathbb{P}(E)$;

2. there exists a section, i.e. a map $\sigma: C \rightarrow S$ such that $\pi \circ \sigma=i d$;

3. $\mathbb{P}(E) \cong \mathbb{P}\left(E^{\prime}\right)$ if and only if there is a holomorphic line bundle L over $C$ such that $E \cong E^{\prime} \otimes L$.

Definition 2.5 A ruled surface $\mathbb{P}(E)$ is decomposable if it has two disjoint sections.

The following Lemma whose proof is in (Maruyama 1970, p. 16) shows the relationship between the ruled surface $S=\mathbb{P}(E)$ and the vector bundle $E$.

Lemma 2.6 There exists a one-to-one correspondance between the line subbundles of $E$ and the sections of $S$. Futhermore, if $\sigma_{L}$ is the section related to the line subbundle L then:

$$
\sigma_{L} \cdot \sigma_{L}=\operatorname{deg} E-2 \operatorname{deg} L
$$

where $\operatorname{deg} E$ is the degree of the determinant bundle of $E$.

Notation 2.7 We recall that the notation $\sigma_{L} \cdot \sigma_{L}$ means the self-intersection of the section $\sigma_{L}$.

Remark 2.8 By Lemma 2.6, $\mathbb{P}(E)$ is decomposable if and only if $E$ is decomposable, i.e. $E=L_{1} \oplus L_{2}$ for line subbundles $L_{i} \hookrightarrow E$.

Consider $\kappa=\min \{\sigma . \sigma, \sigma: C \rightarrow S / \pi \circ \sigma=i d\}$. This number only depends on the ruled surface $S=\mathbb{P}(E)$. Indeed, it does not change when we replace $E$ by $E \otimes L$ for a line bundle $L$ on $C$.

Definition 2.9 The ruled surface $\mathbb{P}(E)$ is stable if $\kappa>0$.

Definition 2.10 A minimal section of $S$ is a section $\sigma: C \rightarrow S$ such that the selfintersection is minimal. That is to say, $\sigma . \sigma=\kappa$. 
Using Lemma 2.6, we notice that a minimal section corresponds to a line subbundle of $E$ with maximal degree. Thus, the invariant $\kappa$ can be written as:

$$
\kappa=\operatorname{deg}(E)-2 \max _{L \hookrightarrow E} \operatorname{deg} L
$$

Now, we are interested in indecomposable ruled surfaces over an elliptic curve. Let $\mathcal{O}_{C}\left(p_{\infty}\right)$ be the line bundle related to the divisor $\left[p_{\infty}\right]$. There are unique nontrivial extensions of invertible sheaves:

$$
0 \longrightarrow \mathcal{O}_{C} \longrightarrow E_{0} \longrightarrow \mathcal{O}_{C} \longrightarrow 0
$$

and

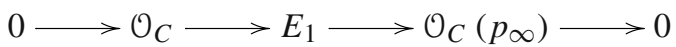

Recall the following theorem of Atiyah as proved in (Atiyah 1955, Th. 6.1):

Theorem 2.11 Up to isomorphism, the unique indecomposable ruled surfaces over $C$ are $S_{0}=\mathbb{P}\left(E_{0}\right)$ and $S_{1}=\mathbb{P}\left(E_{1}\right)$.

Remark 2.12 Equivalenty, any indecomposable vector bundle $E$ of rank 2 on $C$ takes the form $E=E_{i} \otimes L$, for $i=0,1$ and $L$ a line bundle.

As our aim in this paper is the study of the ruled surface $S_{1}$, we will show firstly some important properties of $E_{1}$.

Lemma 2.13 The maximal degree of line subbundles of $E_{1}$ is zero.

Proof Let $L$ be a subbundle of $E_{1}$ and $M$ the quotient line bundle $E_{1} / L$. This gives us the following short exact sequence

$$
0 \longrightarrow L \longrightarrow E_{1} \longrightarrow M \longrightarrow 0
$$

which corresponds to an element in $\operatorname{Ext}^{1}(M, L) \simeq H^{1}\left(M^{-1} \otimes L\right)$. Since the vector bundle $E_{1}$ is indecomposable, this sequence cannot split and hence $H^{1}\left(M^{-1} \otimes L\right) \neq 0$. By Serre duality, this implies that $H^{0}\left(M \otimes L^{-1}\right) \neq 0$ and then $\operatorname{deg}\left(M \otimes L^{-1}\right) \geq 0$, i.e. $\operatorname{deg} M-\operatorname{deg} L \geq 0$. Now from the short exact sequence above we deduce that $\operatorname{deg} E_{1}=\operatorname{deg} M+\operatorname{deg} L$. Hence, we obtain $\operatorname{deg} E_{1}-2 \operatorname{deg} L \geq 0$ and then $\operatorname{deg}(L) \leq 0$ because $\operatorname{deg}\left(E_{1}\right)=1$. Since the trivial line bundle $\mathcal{O}_{C}$ is a line subbundle of $E_{1}$, we have the result.

Remark 2.14 By this Lemma, we can deduce that the ruled surface $S_{1}$ is stable. More precisely, up to isomorphism, it is the unique stable ruled surface over an elliptic curve.

If we consider $\max _{E_{1}}=\left\{L \hookrightarrow E_{1}\right.$, $\left.\operatorname{deg} L=0\right\}$ the set of line subbundles of $E_{1}$ having maximal degree, we have: 
Lemma 2.15 There is a bijection between the jacobian of $C$ and the set $\max _{E_{1}}$ defined by:

$$
\left\{\begin{aligned}
M: \max _{E_{1}} & \longrightarrow J a c(C) \\
L & \longmapsto
\end{aligned}\right.
$$

To prove this Lemma, we have to use a key lemma of Maruyama in (Maruyama 1970, p. 8):

Lemma 2.16 Let $E$ be a vector bundle of rank 2 over a curve. If $L_{1}$ and $L_{2}$ are distinct maximal line subbundles of $E$ such that $L_{1}$ and $L_{2}$ are isomorphic, then $E=L_{1} \oplus L_{2}$.

Now, we can prove Lemma 2.15:

Proof - Let $L_{1}$ and $L_{2}$ be two elements in $\max _{E_{1}}$ such that $L_{1} \cong L_{2}$. We have two possibilities: either $L_{1}=L_{2}$ or they are both distinct. According to Lemma 2.16, the last case cannot occur because $E_{1}$ is not decomposable. Thus, the map $M$ is injective.

- Let $L \in \operatorname{Jac}(C)$ be a line bundle distinct from the trivial bundle. If we apply the functor Hom $(L,--)$ to the exact sequence

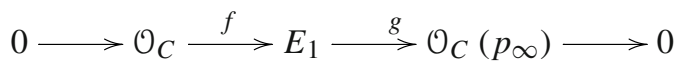

and we use Riemann Roch's theorem, we obtain $\operatorname{dim} \operatorname{Hom}\left(L, E_{1}\right)=1$. There exists a non zero morphism $\tau: L \rightarrow E_{1}$. Thus, if we denote $D$ the effective divisor of zeros of $\tau$ then $L \otimes \mathcal{O}_{C}(D)$ is a line subbundle of $E_{1}$. Since deg $(L)=0, D$ is a effective divisor of zero degree, that is to say $\mathcal{O}_{C}(D)=\mathcal{O}_{C}$. Hence, $L$ is a line subbundle of $E_{1}$.

Remark 2.17 The minimal sections of $S_{1}$ have self-intersection equal to 1 and they are parametrised by the jacobian which is isomorphic to $C$. For any point $\epsilon \in C$, we denote $\sigma_{\epsilon}$ the minimal section corresponding via Lemma 2.15 to the subbundle isomorphic to $\mathcal{O}_{C}\left([\epsilon]-\left[p_{\infty}\right]\right)$.

In the sequel, we use the equivalence between line bundles and divisors on $C$ (Fig. 1).

Lemma 2.18 Let $\sigma_{\epsilon}$ and $\sigma_{\epsilon^{\prime}}$ be two minimal sections of the ruled surface $S_{1}$. If we set $P:=\sigma_{\epsilon} . \sigma_{\epsilon^{\prime}}$, the intersection point of $\sigma_{\epsilon}$ and $\sigma_{\epsilon^{\prime}}$, we have:

$$
\pi(P)=-\epsilon-\epsilon^{\prime}
$$

where $\pi: S_{1} \rightarrow C$ is the projection map and the sign - is the inverse of the law group of $C$.

Proof Let $L_{\epsilon}=\mathcal{O}_{C}\left([\epsilon]-\left[p_{\infty}\right]\right)$ and $L_{\epsilon^{\prime}}=\mathcal{O}_{C}\left(\left[\epsilon^{\prime}\right]-\left[p_{\infty}\right]\right)$ be the subbundles of $E_{1}$ associated with sections $\sigma_{\epsilon}$ and $\sigma_{\epsilon^{\prime}}$ respectively. Using the fact that $S_{1}=\mathbb{P}\left(E_{1}\right)$ we have for every $x \in C, \sigma_{\epsilon}(x)$ and $\sigma_{\epsilon^{\prime}}(x)$ are respectively the projectivisation of the 
Fig. 1 Intersection of two minimal sections on $S_{1}$

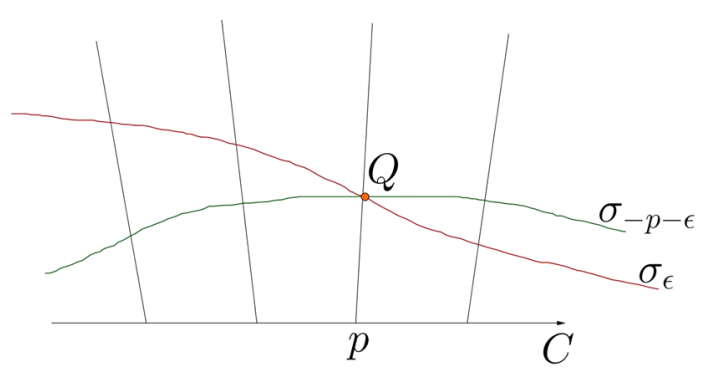

lines $\left(L_{\epsilon}\right)_{x}$ and $\left(L_{\epsilon^{\prime}}\right)_{x}$. Thus, if $P:=\sigma_{\epsilon} \cdot \sigma_{\epsilon^{\prime}}$ is the intersection point of $\sigma_{\epsilon}$ and $\sigma_{\epsilon^{\prime}}$ then the projection $\pi(P)$ is a point on which the line bundles $L_{\epsilon}$ and $L_{\epsilon^{\prime}}$ are colinear. Using the short exact sequences defined by these two line subbundles of $E_{1}$, we can see that $\pi(P)$ is a zero of a global section of the line bundle $I:=\operatorname{det}\left(E_{1}\right) \otimes L_{\epsilon}^{-1} \otimes L_{\epsilon^{\prime}}^{-1}$. As $I$ is a divisor of degree 1 , then we can deduce that the divisor $I$ is equivalent to the divisor $[\pi(P)]$. By definition of the law group of $C$ the divisor $I \sim 3\left[p_{\infty}\right]-[\epsilon]-\left[\epsilon^{\prime}\right]$ is equivalent to the divisor $\left[-\epsilon-\epsilon^{\prime}\right]$. Hence, we have $[\pi(P)] \sim\left[-\epsilon-\epsilon^{\prime}\right]$ and then $\pi(P)=-\epsilon-\epsilon^{\prime}$.

Remark 2.19 Let $Q$ be a point of $S_{1}$ belonging to the fiber $\pi^{-1}(p)$. If the minimal section $\sigma_{\epsilon}$ passes through the point $Q$, then the unique other minimal section passing through the same point $Q$ is the section $\sigma_{-p-\epsilon}$. They might be the same for some $Q$.

We also have the following theorem proved by André Weil in Weil (1995):

Theorem 2.20 A holomorphic vector bundle on a compact Riemann surface is flat if and only if it is the direct sum of indecomposable vector bundles of degree 0 .

By this theorem, the bundle $E_{1}$ is not flat because $\operatorname{deg} E_{1}=1$. However what can we say about its associated ruled surface? The answer of this question is given by Frank Loray and David Marin in Loray and Pérez (2009).

Theorem 2.21 The ruled surface $S_{1}$ has a Riccati foliation $\mathcal{R}$ with irreducible monodromy group $<-z, \frac{1}{z}>$.

Remark 2.22 The generic leaf of the foliation $\mathcal{R}$ is a degree 4 cover of $C$ and three special leaves are covers of degree 2 .

\section{Geometry of the Ruled Surface $S_{1}$}

Let $\pi: S_{1} \rightarrow C$ be the stable ruled surface over $C$ and let $A u t_{C}\left(S_{1}\right)$ be the group of automorphisms of the fibration over the identity of $C$. In the sequel, unless otherwise specified by automorphism group of $S_{1}$ we mean the group $A u t_{C}\left(S_{1}\right)$.

Proposition 3.1 The automorphism group of $S_{1}$ is a group of order 4 which is isomorphic to the 2-torsion group in $C$. 
Proof Let $\psi: S_{1} \rightarrow S_{1}$ be a non trivial automorphism of $S_{1}$. Since the self-intersection is invariant by automorphism, $\psi$ preserves the set of +1 self-intersection sections on $S_{1}$. More precisely, for any $\epsilon \in C$, there exists a unique point $r_{\epsilon} \in C$ such that $\psi\left(\sigma_{\epsilon}\right)=\sigma_{r_{\epsilon}}$. The automorphism $\psi$ induces an automorphism $\widehat{\psi}$ of $C$ such that for any point $\epsilon \in C$ we have $\widehat{\psi}(\epsilon)=r_{\epsilon}$. If we define $C$ as the complex torus $\mathbb{C} / \mathbb{Z}+\tau \mathbb{Z}$, we can write for any $z \in \mathbb{C}, \widehat{\psi}(\hat{z})=a \hat{z}+b$, where $a, b \in \mathbb{C}$ and $a(\mathbb{Z}+\tau \mathbb{Z})=\mathbb{Z}+\tau \mathbb{Z}$.

If we assume this automorphism has a fix point $\epsilon_{0}$, then by definition we have $\psi\left(\sigma_{\epsilon_{0}}\right)=\sigma_{\epsilon_{0}}$. Hence, using the Lemma 2.18, we obtain that for any $p \in C$, $\psi\left(\sigma_{-p-\epsilon_{0}}\right)=\sigma_{-p-\epsilon_{0}}$. For any fiber, the automorphism $\psi$ is Moebius map which fixes at least three points: it is the trivial automorphism, which does not make sense by hypothesis.

Therefore, the automorphism $\widehat{\psi}$ has no fixed points, it is a translation like $\widehat{\psi}(\hat{z})=$ $\hat{z}+b$. As by definition we have: $\widehat{\psi}(-p-\hat{z})=-p-\widehat{\psi}(\hat{z})$, the point $b$ is a point of order 2 of $C$.

Conversely, for any point $p_{i}$ of order 2 on $C$ we can define an automorphism $\Phi_{i}$ on $S_{1}$ such that for any point $p \in C, \Phi_{i}$ restricts to the fiber $\pi^{-1}(p)$ as the unique Moebius map which associates the points of the sections $\left(\sigma_{p_{\infty}}, \sigma_{p_{0}}, \sigma_{p_{1}}, \sigma_{p_{t}}\right)$ to the points of the sections $\left(\sigma_{p_{\infty}+p_{i}}, \sigma_{p_{0}+p_{i}}, \sigma_{p_{1}+p_{i}}, \sigma_{p_{t}+p_{i}}\right)$ respectively. It is defined by:

$$
\left\{\begin{array}{l}
\Phi_{i}: S_{1} \longrightarrow S_{1} \\
P=\sigma_{\epsilon}(p) \longmapsto P^{\prime}=\sigma_{\epsilon+p_{i}}(p)
\end{array}\right.
$$

There exists a one-to-one correspondance between the automorphisms of the fiber bundle $S_{1}$ and the points of order 2 in $C$ which preserves the group structure. Hence we have :

$$
\operatorname{Aut}_{C}\left(S_{1}\right)=\left\{\Phi_{0}, \Phi_{1}, \Phi_{t}, \Phi_{\infty}=I d\right\}
$$

Remark 3.2 If we consider the bijection $\varpi: \epsilon \mapsto \sigma_{\epsilon}$ between the curve $C$ and the minimal sections, we can see the set of minimal sections as a riemann surface in such a way that the application $\varpi$ is holomorphic. Hence we can deduce that the application $\widehat{\psi}=\varpi^{-1} \circ \psi \circ \varpi$ is holomorphic.

Proposition 3.3 The automorphism group of $S_{1}$ preserves the foliation $\mathcal{R}$.

Proof Using the fact that the fundamental group of $C$ is abelian, we can see that

the restriction of the projection $\pi: S_{1} \rightarrow C$ to a generic leaf $F$ of the Riccati foliation $\mathcal{R}$ is a galois covering whose deck transformation group is the restriction of Aut $_{C}\left(S_{1}\right)$ to $F$. Hence the group $A u t_{C}\left(S_{1}\right)$ preserves the Riccati foliation on $S_{1}$.

Corollary 3.4 The Riccati foliation $\mathcal{R}$ is the unique regular Riccati foliation on the ruled surface $S_{1}$.

Proof Let $\mathcal{F}_{1}$ be a smooth Riccati foliation on $S_{1}$. As its monodromy group is an abelian subgroup of $P G L(\mathbb{C}, 2)$, we have three possibilities for its monodromy representation 
- If the conjugacy class of the monodromy is the linear class defined by the group $\langle a z, b z\rangle$, there exists two disjoint invariant sections of $S_{1}$. Hence $S_{1}$ is a ruled surface related to the direct sum of two line bundles over $C$. It does not make sense because $S_{1}$ is indecomposable.

- If the conjugacy class of the monodromy is the euclidian class defined by the group $\langle z+1, z+s\rangle$, there exists an invariant section on $S_{1}$ with zero self-intersection. In fact by the theorem of Camacho Sad (see Camacho and Neto 1985), any invariant curve of regular foliation has a zero self-intersection. This monodromy representation does not make sense in $S_{1}$ because we have $\min \left\{\sigma . \sigma, \sigma: C \rightarrow S_{1} / \pi \circ \sigma=i d\right\}=1$.

The only remaining possibility is that the monodromy has image the group $<-z, \frac{1}{z}>$. Thus, the foliation $\mathcal{F}_{1}$ is conjugated to $\mathcal{R}$ by an element in $A u t_{C}\left(S_{1}\right)$. As this automorphism group of the fibration $S_{1}$ preserves the foliation $\mathcal{R}$, we have $\mathcal{R}=\mathcal{F}_{1}$.

Lemma 3.5 With the identification $\operatorname{Jac}(C) \bumpeq C$ given by Lemma 2.1 and the notation considered in Remark 2.17 we have: There exists a ramified double cover of the ruled surface $S_{1}$ defined by the map :

$$
\left\{\begin{array}{ccc}
\varphi: C \times J a c(C) & \longrightarrow & S_{1} \\
(p, \epsilon) & \longmapsto P=\sigma_{\epsilon}(p)
\end{array}\right.
$$

such that its involution is defined by:

$$
\left\{\begin{aligned}
\mathrm{i}: C \times J a c(C) & \longrightarrow C \times \operatorname{Jac}(C) \\
(p, \epsilon) & \longmapsto(p,-p-\epsilon)
\end{aligned}\right.
$$

Proof According to Lemma 2.18, three minimal sections cannot meet at the same point, then we deduce that for any $p \in C$, the morphism

$$
\left\{\begin{array}{cl}
\varphi_{p}: J a c(C) & \longrightarrow \pi^{-1}(p) \\
\epsilon & \longmapsto \sigma_{\epsilon}(p)
\end{array}\right.
$$

is not constant: it is a ramified cover between Riemann surfaces. Futhermore, by Remark 2.19, we know that at most two minimal sections can pass through a given point, then the map $\varphi$ is a ramified double cover.

The immediate consequence of this Lemma is the following :

Theorem 3.6 There exists a irreducible singular holomorphic 2-web $\mathcal{W}$ on $S_{1}$ defined by the minimal sections whose discriminant $\Delta$ is a leaf of the foliation $\mathcal{R}$.

Remark 3.7 The 2-web $\mathcal{W}$ defined by minimal sections cannot be decomposed because there exists a unique smooth Riccati foliation on $S_{1}$.

Proof By Lemma 3.5, for any point $P \in \pi^{-1}(p)$ there exists a minimal section $\sigma_{\epsilon}$ passing through this point. Likewise, by Lemma 2.18, unless they are equal, the 
minimal section $\sigma_{-p-\epsilon}$ intersects transversally the minimal section $\sigma_{\epsilon}$ at the point $P$. As the sections $\sigma_{\epsilon}$ and $\sigma_{-p-\epsilon}$ are distinct if and only if $2 \epsilon \neq-p$, we deduce that there exists a singular holomorphic 2 -web on $S_{1}$ such that its discriminant is defined by :

$$
\Delta=\cup_{p \in C}\left\{P \in \pi^{-1}(p) / P=\sigma_{\epsilon}(p), 2 \epsilon=-p\right\}
$$

In order to prove that $\Delta$ is a leaf of the Riccati, we need the following :

Lemma 3.8 There exists a linear foliation $\mathcal{F}$ on $C \times J a c(C)$ such that $\varphi_{*} \mathcal{F}=\mathcal{R}$.

Proof Assume that $C \times \operatorname{Jac}(C) \simeq(\mathbb{C} / \mathbb{Z}+\tau \mathbb{Z}) \times(\mathbb{C} / \mathbb{Z}+\tau \mathbb{Z})$. If we consider the linear foliation $\widetilde{\mathcal{F}}:=\mathrm{d} x+2 \mathrm{~d} y$ on $\mathbb{C}_{x} \times \mathbb{C}_{y}$, then $\widetilde{\mathcal{F}}$ is invariant by the action of the lattice $\mathbb{Z}+\tau \mathbb{Z}$. Thus we can project the foliation $\widetilde{\mathcal{F}}$ to a foliation, $\mathcal{F}$ on $C \times \operatorname{Jac}(C)$ such that the monodromy is defined by :

$$
\left\{\begin{aligned}
\xi: \Lambda & \longrightarrow \operatorname{Aut}(C) \\
\lambda & \longmapsto p \mapsto p-\frac{1}{2} \lambda
\end{aligned}\right.
$$

where $\Lambda$ is the lattice $\mathbb{Z}+\tau \mathbb{Z}$.

The foliation $\mathcal{F}$ is transverse to the first projection on $C \times J a c(C)$ with a monodromy group isomorphic to the group of points of order $2\left\{p_{\infty}, p_{0}, p_{1}, p_{t}\right\}$. Moreover, if $\mathcal{F}_{(p, \epsilon)}$ is the leaf passing through the point $(p, \epsilon)$, then by definition we have :

$$
\mathrm{i}\left(\mathcal{F}_{(p, \epsilon)}\right)=\mathcal{F}_{(p,-p-\epsilon)}
$$

where $i$ is the involution of the ramified double cover $\varphi$. Hence, $\varphi_{*} \mathcal{F}$ the direct image of the foliation $\mathcal{F}$ by $\varphi$ is a Riccati foliation on $S_{1}$ having the same monodromy group than $\mathcal{R}$. Using the uniqueness of $\mathcal{R}$ by Corollary 3.4, we obtain the result.

As by definition the curve $G=\{(2 p,-p) / p \in C\}$ is a leaf of the foliation $\mathcal{F}$, using the foregoing lemma we can deduce that $\varphi(G)=\Delta$ is a leaf of $\mathcal{R}$. Which completes the proof of Theorem 3.6.

\subsection{Study of Special Leaves of the Riccati Foliation $\mathcal{R}$}

According to Lemma 3.8, if $P^{\prime}=\sigma_{\epsilon^{\prime}}\left(p^{\prime}\right) \in S_{1}$ then the Riccati leaf passing through at this point is given by

$$
\mathcal{R}_{P^{\prime}}=\left\{P \in S_{1} \mid P=\sigma_{\epsilon}(p), p=\pi(P), 2 \epsilon=2 \epsilon^{\prime}+p^{\prime}-p\right\}
$$

Thus, if we use this characterisation of the Riccati leaves on $S_{1}$, we have the following lemma :

Lemma 3.9 There exists three special leaves $\mathcal{R}_{0}, \mathcal{R}_{1}$ and $\mathcal{R}_{t}$ of the foliation $\mathcal{R}$ which are double cover of $C$. More precisely, they are respectively the set of fixed points of the automorphisms $\Phi_{0}, \Phi_{1}$ and $\Phi_{t}$. 
Proof We just give the proof for the leaf $\mathcal{R}_{0}$ because it is the same process for the other special leaves.

- Let $\mathcal{R}_{0}$ be the Riccati leaf passing through the point $P_{0}=\sigma_{p_{0}}\left(p_{0}\right)$, then by definition, we have :

$$
\mathcal{R}_{0}=\left\{P \in S_{1} \mid P=\sigma_{\epsilon}(p), p=\pi(P), 2 \epsilon=p_{0}-p\right\}
$$

According to the monodromy of $\mathcal{R}$, if the leaf $\mathcal{R}_{0}$ passes through the point $P=$ $\sigma_{\epsilon}(p)$ then it passes through the points $\sigma_{\epsilon+p_{i}}(p)$, where $p_{i}$ is a 2-torsion point of $C$. Since $2 \epsilon=p_{0}-p$, we deduce from Lemma 2.18 that: $\sigma_{\epsilon+p_{0}}(p)=\sigma_{\epsilon}(p)$ and $\sigma_{\epsilon+p_{1}}(p)=\sigma_{\epsilon+p_{t}}(p)$ thus, Ric $c_{0}$ meets any fiber of $S_{1}$ twice. It is a double cover over $C$.

- Let $\Phi_{0}$ be the automorphism of $S_{1}$ related to the point $p_{0}$ defined by:

$$
\left\{\begin{array}{l}
\Phi_{0}: S_{1} \longrightarrow \quad S_{1} \\
P=\sigma_{\epsilon}(p) \longmapsto P^{\prime}=\sigma_{\epsilon+p_{0}}(p)
\end{array}\right.
$$

and consider the set of its fix points Fix $0=\left\{P / \Phi_{0}(P)=P\right\}$.

If $P=\sigma_{\epsilon}(p)$ is the fix point of $\Phi_{0}$, then by Lemma 2.18 , we have $2 \epsilon=p_{0}-p$ and therefore $P \in \mathcal{R}_{0}$. Conversely, if $P \in \mathcal{R}_{0}$, we have by definition $2 \epsilon=p_{0}-p$, and according to Lemma 2.18, we have $\sigma_{\epsilon+p_{0}}(p)=\sigma_{\epsilon}(p)$. Thus, we deduce that:

$$
\mathcal{R}_{0}=\left\{P / \Phi_{0}(P)=P\right\}
$$

According to all the foregoing, we have:

Remark 3.10 The 2-web given by the +1 self-intersection sections, the Riccati foliation and the $\mathbb{P}^{1}$-bundle $\pi: S_{1} \rightarrow C$ form a singular holomorphic 4-web $\mathbf{W}$ on $S_{1}$ whose the discriminant is $\Delta$.

\subsection{The Geometry of the 4-Web W}

Let $(x, y)$ be the local coordinates of $\mathbb{C}^{2}$. As the linear foliations $\mathcal{G}$ and $\mathcal{H}$ respectively defined by $\mathrm{d} y=0$ and by $\mathrm{d} y+\mathrm{d} x=0$ are invariant by the action of the lattice $\mathbb{Z}+\tau \mathbb{Z}$, we can project them to a decomposable 2 -web $\mathcal{W}^{\prime}$ on $C \times J a c(C)$.

Proposition 3.11 The direct image $\varphi_{*}\left(\mathcal{W}^{\prime}\right)$ of the 2-web $\mathcal{W}^{\prime}$ by the ramified cover $\varphi$ is the 2-web $\mathcal{W}$ on $S_{1}$ defined by the minimal sections.

Proof As by definition the 2 -web $\mathcal{W}^{\prime}$ is invariant by the involution of the ramified double cover $\varphi$, its direct image is also a singular holomorphic 2-web on $S_{1}$. Let $\left(p^{\prime}, \epsilon^{\prime}\right) \in C \times \operatorname{Jac}(C)$ and consider

- $A_{\epsilon^{\prime}}=\left\{(p, \epsilon) \in C \times \operatorname{Jac}(C) / \epsilon=\epsilon^{\prime}\right\}$ 
- $B_{\epsilon^{\prime}}=\left\{(p, \epsilon) \in C \times \operatorname{Jac}(C) / \epsilon=-p+\left(p^{\prime}+\epsilon^{\prime}\right)\right\}$,

the leaves of the 2-web $\mathcal{W}^{\prime}$ passing through this point. Since, using Lemma 2.18, we have: $\varphi\left(A_{\epsilon^{\prime}}\right)=\sigma_{\epsilon^{\prime}}$ and $\varphi\left(B_{\epsilon^{\prime}}\right)=\sigma_{-p^{\prime}-\epsilon^{\prime}}$, then the leaves of $\varphi_{*}\left(\mathcal{W}^{\prime}\right)$ are the minimal sections of $S_{1}$ which are the same along the discriminant $\Delta$.

The local study of the 4-web $\mathbf{W}$ on $S_{1}$ is the same as the 4-web on $C \times J a c(C)$ given by the 2 -web $\mathcal{W}^{\prime}$, the foliation $\mathcal{F}$ and the $\operatorname{Jac}(C)$-bundle defined by the first projection on $C \times \operatorname{Jac}(C)$.

Theorem 3.12 Outside the discriminant locus $\Delta$, the 4-web $\boldsymbol{W}$ is locally parallelizable.

Proof According to the foregoing, the pull-back of 4 -web $\mathbf{W}$ by the ramified cover $\varphi$ is locally the 4 -web defined by $W(x, y, y+x, y+2 x)$ on $\mathbb{C}^{2}$. It is a holomorphic parallelizable web.

Remark 3.13 An immediate consequence of Theorem 3.12 is that the curvature of the 4-web $\mathbf{W}$ is zero.

The second part of this paper aims to use the theory of birational geometry in order to find the theoretic results of the first part by computations on the birational trivialisation $C \times \mathbb{P}^{1}$.

\section{Geometry of 4-Web W After Elementary Transformations}

Let $\pi: S_{1} \rightarrow C$ be the $\mathbb{P}^{1}$-bundle and $\left\{p_{0}, p_{1}, p_{t}, p_{\infty}\right\}$ the set of points of order 2 in $C$.

Definition 4.1 An elementary transformation at the point $P \in \pi^{-1}(p)$ is the birational map given by the composition of the blow-up of the point $P$, followed by the contraction of the proper transform of the fiber $\pi^{-1}(p)$.

Remark 4.2 After elementary transformation at the point $P$, we obtain a new ruled surface with a point $\widetilde{P}$ which is the contraction of the proper transform of the fiber $\pi^{-1}(p)$.

How many elementary transformations do you need to trivialize the ruled surface $S_{1}$ ?

Lemma 4.3 The ruled surface $S_{1}$ is obtained after three elementary transformations at the points $\widetilde{P}_{0}=\left(p_{0}, 0\right), \widetilde{P}_{1}=\left(p_{1}, 1\right)$ and $\widetilde{P}_{\infty}=\left(p_{\infty}, \infty\right)$ on the trivial bundle $C \times \mathbb{P}^{1}$.

In fact, if we perform the elementary transformations at the three special points $\widetilde{P}_{0}$, $\widetilde{P}_{1}$ and $\widetilde{P}_{\infty}$ of $C \times \mathbb{P}^{1}:$ (see Fig. 2), we have a ruled surface $S$ with three special points $P_{0}, P_{1}$ and $P_{\infty}$ (see Fig. 3).

Recall that after 3 elementary transformations, if $\sigma$ is a section on $S$ such that $\sigma^{\prime}$ is its strict transform on the trivial bundle, we have: $\sigma . \sigma=\sigma^{\prime} . \sigma^{\prime}+r$ where $r=\epsilon_{0}+\epsilon_{1}+\epsilon_{\infty}$ such that

$$
\epsilon_{i}=\left\{\begin{array}{l}
-1 \text { if } P_{i} \in \sigma \\
+1 \text { if } P_{i} \notin \sigma
\end{array}\right.
$$


Fig. 2 Special points of the trivial $\mathbb{P}^{1}$-bundle over $C$
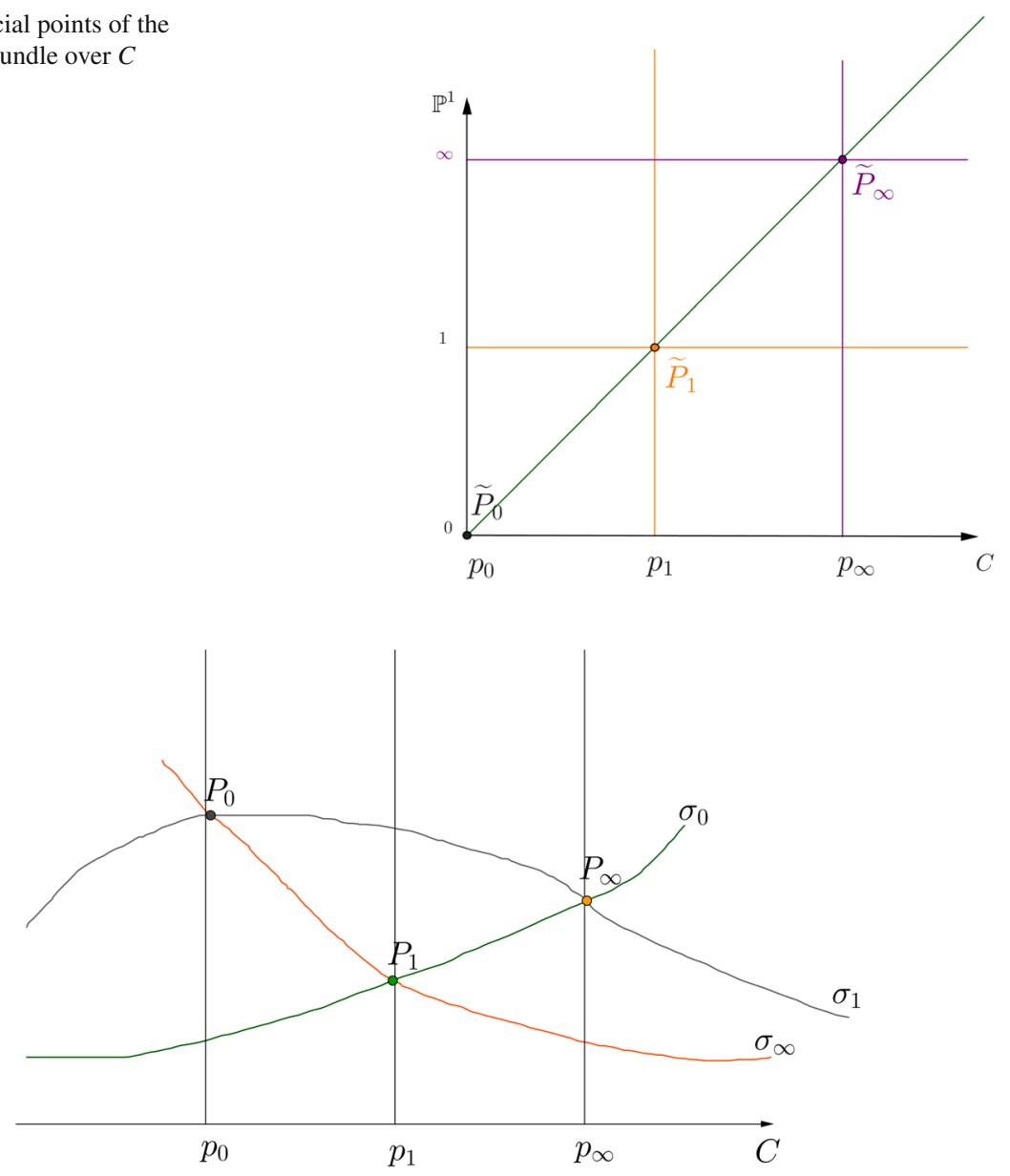

Fig. 3 Special points and special sections of +1 self-intersection of $S_{1}$

in particular, $r \in\{-1,1,-3,3\}$. Then, we can deduce that the ruled surface $S$ has a invariant $\kappa \leq 1$.

1. If $\kappa=0$, there is a section $\sigma$ of zero self-intersection on $S$ and then its strict transform $\sigma^{\prime}$ has odd self-intersection. This cannot hold because all the sections of the trivial bundle have even self-intersection;

2. If $\kappa<0$, let $\sigma$ be a section of $S$ such that $\sigma \cdot \sigma=\kappa$. Using the fact that its strict transform $\tilde{\sigma}$ has a strictly positive self-intersection, we can see that either $\kappa=-1$ or $\kappa=-2$. As the ruled surface $S$ admits a section $\sigma_{0}$ of +1 self-intersection, the case where $\kappa=-2$ cannot occur. If $\kappa=-1$, then $\widetilde{\sigma}$ is a section of +2 self-intersection on $C \times \mathbb{P}^{1}$ passing through by three points $\widetilde{P}_{0}, \widetilde{P}_{1}$ and $\widetilde{P}_{\infty}$. Thus, this section defines a non-constant morphism $\widetilde{\sigma}: C \rightarrow \mathbb{P}^{1}$ of degree 1 . It is absurd because the curve $C$ is not rational. 
According to these two cases, after three elementary transformations on the trivial bundle $C \times \mathbb{P}^{1}$, we obtain a ruled surface such that its invariant $\kappa=1$. Therefore, it is the stable ruled surface over an elliptic curve.

\subsection{The Riccati Foliation on $S_{1}$ After Elementary Transformations}

Proposition 4.4 After elementary transformations of the three special points $P_{0}, P_{1}$ and $P_{\infty}$ on $S_{1}$, the Riccati foliation $\mathcal{R}$ induces a Riccati foliation $\widetilde{\mathcal{R}}$ on the trivial bundle $C \times \mathbb{P}^{1}$ such that the points $\widetilde{P}_{0}=\left(p_{0}, 0\right), \widetilde{P}_{1}=\left(p_{1}, 1\right)$ and $\widetilde{P}_{\infty}=\left(p_{\infty}, \infty\right)$ are radial singularities.

If $((x, y), z)$ are cordinates of the trivial bundle $C \times \mathbb{P}^{1}$, then the foliation $\widetilde{\mathcal{R}}$ is defined by the 1 -form $d z-\left[a(x, y) z^{2}+b(x, y) z+c(x, y)\right] \frac{d x}{2 y}$ where $a, b, c$ are the meromorphic functions with pole of order 1 at the points $p_{0}, p_{1}$ and $p_{\infty}$, i.e. $a, b, c \in H^{0}\left(\mathcal{O}_{C}\left(\left[p_{0}\right]+\left[p_{1}\right]+\left[p_{\infty}\right]\right)\right)$. Recall that, if we set the effective divisor $D=\left[p_{0}\right]+\left[p_{1}\right]+\left[p_{\infty}\right]$, we have $H^{0}\left(\mathcal{O}_{C}(D)\right)=\{F \in \mathcal{M}(C), \operatorname{div}(F) \geq-D\}$ where $\mathcal{M}(C)$ is the field of meromorphic functions on $C$. By Puiseux parametrisation of the curve $C$ we can see that meromorphic functions $\frac{1}{y}$ and $\frac{x}{y}$ belong to $H^{0}\left(\mathcal{O}_{C}(D)\right)$, and then we can deduce that the family $\left\{1, \frac{1}{y}, \frac{x}{y}\right\}$ is a free in $H^{0}\left(\mathcal{O}_{C}(D)\right)$. Hence, as by Riemann-Roch theorem $H^{0}\left(\mathcal{O}_{C}(D)\right)$ is a vector space of dimension 3, we have:

$$
H^{0}\left(\mathcal{O}_{C}\left(\left[p_{0}\right]+\left[p_{1}\right]+\left[p_{\infty}\right]\right)\right)=\mathbb{C}<1, \frac{1}{y}, \frac{x}{y}>
$$

Thus, it means that $a=\frac{a_{0}+a_{1} x+a_{2} y}{y}$, where $a_{i}$ are constant. If we write the same relation for the functions $b$ and $c$, we obtain that the foliation $\widetilde{\mathcal{R}}$ is defined by the following 1-form:

$$
y d z-\left[\left(a_{0}+a_{1} x+a_{2} y\right) z^{2}+\left(b_{0}+b_{1} x+b_{2} y\right) z+\left(c_{0}+c_{1} x+c_{2} y\right)\right] \frac{d x}{2 y}
$$

As the foliation is invariant by the involution $I:(x, y) \mapsto(x,-y)$ on $C$, the coefficients $a_{2}, b_{2}$, and $c_{2}$ are zero. Futhermore, if we use the relation on an elliptic curve, $y^{2}=x(x-1)(x-t)$, and the fact that the points $(0,0,0),(1,0,1),\left(p_{\infty}, \infty\right)$ are the radial singularities, we have $\widetilde{\mathcal{R}}$ is defined by the 1 -form :

$$
w:=d z+\left[\frac{-z^{2}}{4 x(x-1)}-\frac{z}{2 x}+\frac{1}{4(x-1)}\right] d x
$$


Proposition 4.5 The monodromy group of the foliation $\widetilde{\mathcal{R}}$ along a generic fiber $\pi^{-1}\left(x_{0}, y_{0}\right)$ is an abelian group given by these automorphisms: $\widetilde{\Phi}_{0}: z \mapsto \frac{z-x_{0}}{z-1}$, $\widetilde{\Phi}_{1}: z \mapsto \frac{x_{0}}{z}, \widetilde{\Phi}_{t}: z \mapsto \frac{x_{0}(z-1)}{z-x_{0}}, \widetilde{\Phi}_{\infty}: z \mapsto z$

Proof Let $\widetilde{\sigma}_{\infty}:=\{z=\infty\}, \widetilde{\sigma}_{0}:=\{z=0\}, \widetilde{\sigma}_{1}:=\{z=1\}$ and $\widetilde{\sigma}_{d}:=\{z=x\}$ be the four special sections obtained after elementary transformations. By definition of the monodromy group of $\mathcal{R}$, we can see that for the point $p_{0}$ of order 2 , the automorphism $\widetilde{\Phi}_{0}$ restricted to any fiber is the unique Moebius transformation which sends respectively the points of the sections $\left(\widetilde{\sigma}_{0}, \widetilde{\sigma}_{1}, \widetilde{\sigma}_{\infty}, \widetilde{\sigma}_{d}\right)$ to the points of the sections $\left(\widetilde{\sigma}_{d}, \widetilde{\sigma}_{\infty}, \widetilde{\sigma}_{1}, \widetilde{\sigma}_{0}\right)$. Using the same process for the other automorphisms, we obtain the result.

We can also describe the special leaves of the foliation $\widetilde{\mathcal{R}}$. In fact, if we consider $\phi_{i}: C \times \mathbb{P}^{1} \rightarrow C \times \mathbb{P}^{1} ;(x, y, z) \mapsto\left(x, y, \widetilde{\Phi_{i}}(z)\right)$, then according to Lemma 3.9 , the special leaves are defined by :

1. $\widetilde{\mathcal{R}}_{0}:=\left\{(x, y, z), \phi_{0}(x, y, z)=(x, y, z)\right\}=\left\{(x, z),-z^{2}-x+2 z=0\right\}$

2. $\widetilde{\mathcal{R}}_{1}:=\left\{(x, y, z), \phi_{1}(x, y, z)=(x, y, z)\right\}=\left\{(x, z),-z^{2}+x=0\right\}$

3. $\widetilde{\mathcal{R}}_{t}:=\left\{(x, y, z), \phi_{t}(x, y, z)=(x, y, z)\right\}=\left\{(x, z), z^{2}-2 x z+x=0\right\}$

Now it is natural to ask if we can find the expression of the generic leaf of $\widetilde{\mathcal{R}}$. To do this, we use the special leaves to find a first integral. Let

$$
f_{0}:=-z^{2}+2 z-x, \quad f_{1}:=-z^{2}+x, \quad f_{t}:=z^{2}-2 x z+x
$$

be the polynomials which define respectively the leaves $\widetilde{\mathcal{R}}_{0}, \widetilde{\mathcal{R}}_{1}, \widetilde{\mathcal{R}}_{t}$ and consider the function $\gamma: C_{x} \times \mathbb{P}_{z}^{1} \rightarrow C_{x} \times \mathbb{P}_{y}^{1} ;(x, z) \mapsto(x, F(x, z))$, where

$$
F(x, z)=x\left(\frac{f_{0}}{f_{t}}\right)^{2}
$$

The pull-back $\gamma^{*} d y$ of the 1 -form $d y$ by $\gamma$ is a foliation on $C_{x} \times \mathbb{P}_{z}^{1}$ having the function $F(x, z)$ as a first integral and such that the curves $\widetilde{\mathcal{R}}_{0}, \widetilde{\mathcal{R}}_{1}$ and $\widetilde{\mathcal{R}}_{t}$ are invariant. Hence, the foliation $\gamma^{*} d y$ coincide with the Riccati foliation $\widetilde{\mathcal{R}}$. Thus, we can deduce that :

Lemma 4.6 The foliation $\widetilde{\mathcal{R}}$ on $C \times \mathbb{P}^{1}$ has a rational first integral defined by the following function :

$$
F(x, z)=\frac{x\left(z^{2}-2 z+x\right)^{2}}{\left(-z^{2}+2 x z-x\right)^{2}}
$$

\subsection{The 2-Web After Elementary Transformations}

After elementary transformations at the three special points on $S_{1}$, the generic +1 self-intersection sections (i.e not passing through the three special points) becomes a 
Fig. 4 Generic +4 self-intersection section

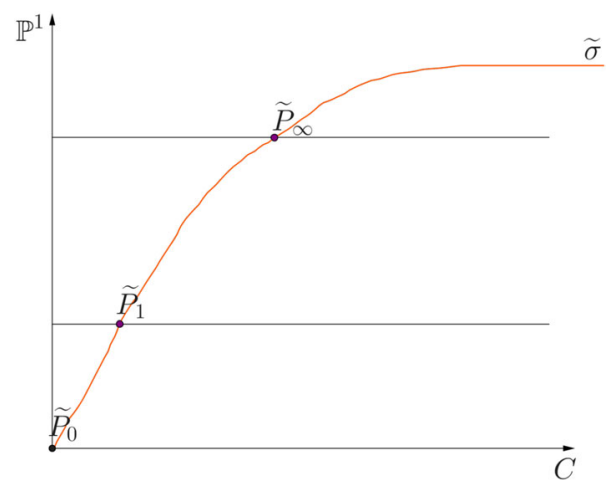

+4 self-intersection sections of $C \times \mathbb{P}^{1}$ passing through the points $(0,0,0),(1,0,1)$ and $\left(p_{\infty}, \infty\right)$ : see Fig. 4 .

Lemma $4.7 A+4$ self-intersection section passing through the points $\widetilde{P}_{0}, \widetilde{P}_{1}$ and $\widetilde{P}_{\infty}$ is either given by a graph $z=\frac{\left(1-a_{0}\right)\left(b_{0} x-a_{0} y\right)}{b_{0}\left(x-a_{0}\right)}$, or a graph $z=x$.

Proof If $\sigma: C \rightarrow \mathbb{P}^{1}$ is a +4 self-intersection section on the trivial bundle, then it defines a rational map of degree 2 generated by two sections $\sigma_{1}$ and $\sigma_{2}$ of a line bundle of degree 2 over $C$; more precisely, for any point $(x, y) \in C, \sigma(x, y)=$ $\left(\sigma_{1}(x, y): \sigma_{2}(x, y)\right)$. Since for any line bundle of degree 2 over $C$, there exists a point $p=\left(a_{0}, b_{0}\right) \in C$ such that $L=[p]+\left[p_{\infty}\right]$, we have two cases:

- if $p \neq p_{\infty}$, due to the Riemann Roch's theorem, we have the vector space $H^{0}(L)=\mathbb{C}\left\langle y-b_{0}, x-a_{0}\right\rangle$ and then, $\sigma$ is a graph given by

$$
z=\frac{a\left(y-b_{0}\right)+b\left(x-a_{0}\right)}{c\left(y-b_{0}\right)+d\left(x-a_{0}\right)}, \quad a, b, c, d \in C
$$

Using the fact that the section passes through the points $\widetilde{P}_{0}, \widetilde{P}_{1}$ and $\widetilde{P}_{\infty}$ and the Puiseux parametrisation of elliptic curve at the infinity point is given by $\varepsilon \mapsto\left(\frac{1}{\varepsilon^{2}}, \frac{1}{\varepsilon^{3}}\right)$, we obtain a system of equations which solutions are $\left\{a=d \frac{a_{0}\left(a_{0}-1\right)}{b_{0}}, b=-d\left(a_{0}-1\right), c=0, d=d\right\}$ where $d \neq 0$;

- if $p=p_{\infty}$, then we have $H^{0}(L)=\mathbb{C}<1, \frac{1}{x}>$, likewise using the fact that the section passes through the points $(0,0,0),(1,0,1)$ and $\left(p_{\infty}, \infty\right)$, we obtain that $\sigma$ is the graph $z=x$.

From now on, we denote $\mathcal{S}$ the set of +4 self-intersection sections of the trivial bundle which pass through the points $\widetilde{P}_{0}, \widetilde{P}_{1}$ and $\widetilde{P}_{\infty}$. 
Proposition 4.8 For any point $(u, v, z) \in C \times \mathbb{P}^{1}$ such that $v \neq 0$, there exists $a+4$ self-intersection section in $\mathcal{S}$ which passes through this point.

Proof Let $(u, v, z) \in C \times \mathbb{P}^{1}$ such that $v \neq 0$, we have to find the points $\left(a_{0}, b_{0}\right) \neq$ $(u, v)$ of $C$ such that $z=\frac{\left(1-a_{0}\right)\left(b_{0} u-a_{0} v\right)}{b_{0}\left(u-a_{0}\right)}$.

Using the fact that $b_{0}^{2}=a_{0}\left(a_{0}-1\right)\left(a_{0}-t\right)$ and $v^{2}=u(u-1)(u-t)$, we have the following equation:

(\$) : $(u-z)^{2} a_{0}^{3}-\left[(2(u z-u))(-z+u)-(-z+u)^{2} t-v^{2}\right] a_{0}^{2}+\left[(u z-u)^{2}-\right.$ $\left.(2(u z-u))(-z+u) t+v^{2}\right] a_{0}-(u z-u)^{2} t=0$

1. if $(u-z)=0$, then $(\boldsymbol{\beta})$ becomes $\left(a_{0}-u\right)\left(a_{0}-\frac{t(u-1)}{u-t}\right)=0$. As by hypothesis $v \neq 0$, we obtain two solutions given by the point $\left(a_{0}, b_{0}\right)$ such that $a_{0}=\frac{t(u-1)}{u-t}$ and the point $p_{\infty}$;

2. if $(u-z) \neq 0$, then the solutions verify the following second degree equation:

$$
(\star):(u-z)^{2} a_{0}^{2}+\left[(-t-u) z^{2}+2 u(t+1) z-u(t+u)\right] a_{0}+t u(z-1)^{2}=0
$$

The +4 self-intersection sections in $\mathcal{S}$ define a singular holomorphic 2 -web $\mathcal{W}$ on $C \times \mathbb{P}^{1}$ such that the discriminant is the union of $\Delta$, the discriminant of the equation $(\star)$ and the singular fibers at the points $p_{0}, p_{1}$ and $p_{\infty}$. We have:

$$
\left.\Delta:=(t-u) z^{4}-4(t-1)\right) u z^{3}+2 u(2 t u+t-u-2) z^{2}-4 u^{2}(t-1) z+u^{2}(t-u)=0
$$

Lemma 4.9 The discriminant $\Delta$ is a leaf of order 4 of the Riccati foliation $\widetilde{\mathcal{R}}$.

Proof In fact, by the definition of the first integral of the foliation $\widetilde{\mathcal{R}}$, we have:

$$
F(x, z)-t=-\frac{\left.(t-u) z^{4}-4(t-1)\right) u z^{3}+2 u(2 t u+t-u-2) z^{2}-4 u^{2}(t-1) z+u^{2}(t-u)}{\left(2 x z-z^{2}-x\right)^{2}}
$$

Therefore, the first integral is constant along of the discriminant $\Delta$.

According to the foregoing, on the birational trivialisation of $S_{1}$, we have a 4 -web $\mathcal{W}_{4}$ defined by the 2 -web $\mathcal{W}$, the Riccati foliation $\widetilde{\mathcal{R}}$ and the trivial fiber bundle.

\subsection{Geometry of the 4-web $\mathcal{W}_{4}$}

We want to find the slopes of the leaves of $\mathcal{W}_{4}$ in order to represent it by a differential equation. Let $\left(x_{0}, y_{0}, z_{0}\right) \in C \times \mathbb{P}^{1}$ be a generic point. As the leaves of the 2 -web $\mathcal{W}$ passing through this point are respectively the graph $z=\frac{\left(1-a_{0}\right)\left(b_{0} x-a_{0} y\right)}{b_{0}\left(x-a_{0}\right)}$ 
and $z=\frac{\left(1-a_{0}{ }^{\prime}\right)\left(b_{0}{ }^{\prime} x-a_{0}{ }^{\prime} y\right)}{b_{0}{ }^{\prime}\left(x-a_{0}{ }^{\prime}\right)}$ such that the points $\left(a_{0}, b_{0}\right)$ and $\left(a_{0}{ }^{\prime}, b_{0}{ }^{\prime}\right)$ verify the equation $(\star)$, we deduce that their slopes at the point $\left(x_{0}, y_{0}, z_{0}\right)$ are respectively given by the following formulas:
1. $Z_{1}=\frac{1-a_{0}-z_{0}}{x_{0}-a_{0}}+\left(z_{0}+\frac{\left(a_{0}-1\right) x_{0}}{x_{0}-a_{0}}\right)\left(\frac{3 x_{0}^{2}-2(1+t) x_{0}+t}{2 x_{0}\left(x_{0}-1\right)\left(x_{0}-t\right)}\right)$;
2. $Z_{2}=\frac{1-a_{0}{ }^{\prime}-z_{0}}{x_{0}-a_{0}^{\prime}}+\left(z_{0}+\frac{\left(a_{0}{ }^{\prime}-1\right) x_{0}}{x_{0}-a_{0}^{\prime}}\right)\left(\frac{3 x_{0}^{2}-2(1+t) x_{0}+t}{2 x_{0}\left(x_{0}-1\right)\left(x_{0}-t\right)}\right)$.

Thus, the irreducible 2 -web $\mathcal{W}$ is defined by the following differential equation:

$$
\left(\frac{\mathrm{d} z}{\mathrm{~d} x}\right)^{2}-\left(\frac{z^{2}+2(x-1) z-x}{2 x(x-1)}\right) \frac{\mathrm{d} z}{\mathrm{~d} x}+\frac{z(z-1)\left(\left(2 t x-x^{2}-t\right) z-x^{3}+x^{2}-t x+2\right)}{4 x^{2}(x-1)^{2}(t-x)}
$$

Futhermore, if we consider

$$
Z_{0}=\frac{1}{4}\left[\frac{\left(z_{0}^{2}+2\left(x_{0}-1\right) z_{0}-x_{0}\right)}{x_{0}\left(x_{0}-1\right)}\right]
$$

the slope of the foliation $\widetilde{\mathcal{R}}$ at the point $\left(x_{0}, y_{0}, z_{0}\right)$, then the 4-web $\mathcal{W}_{4}$ is locally equivalent to the 4 -web on the complex plane given by $\mathcal{W}\left(\infty, Z_{0}, Z_{1}, Z_{2}\right)$.

Theorem 4.10 The 4-web $\mathcal{W}\left(\infty, Z_{0}, Z_{1}, Z_{2}\right)$ is locally equivalent to a parallelizable 4-web.

Proof The pull-back of the foliation $\widetilde{\mathcal{R}}$ by the multiplication of order 2 on $C$ is another Riccati foliation $\widetilde{\mathcal{R}}_{2}$ on $C \times \mathbb{P}^{1}$ with trivial monodromy.

Let $M_{2}: C \rightarrow C$, be the multiplication of order 2 on $C$ then, for any point $(x, y) \in$ $C$ the first projection of $M_{2}(x, y)$ is given by the following formula :

$$
p r_{1} \circ M_{2}(x, y)=\frac{\left(3 x^{2}-2(t+1) x+t\right)^{2}}{4 x(x-1)(x-t)}+(1+t)-2 x
$$

Using the pull-back of the special leaves, we can choose three curves by:

1. $C_{0}:=\left\{(x, y, z), z=z_{0}=\frac{-x^{2}+t}{2(t-x)}\right\}$
2. $C_{1}:=\left\{(x, y, z), z=z_{1}=\frac{\left(-x^{2}+t\right)}{2 y}\right\}$
3. $C_{2}:=\left\{(x, y, z), z=z_{2}=-\frac{\left(-x^{2}+t\right)}{2 y}\right\}$

which are the leaves of $\widetilde{\mathcal{R}}_{2}$. Now, if we consider the map $\psi: C \times \mathbb{P}^{1} \rightarrow C \times \mathbb{P}^{1}$ which for any coordinate $(X, Z)$ relates:

$$
\psi(X, Z)=\left(\frac{\left(3 X^{2}-2(t+1) X+t\right)^{2}}{4 X(X-1)(X-t)}+(1+t)-2 X, \frac{Z \mu z_{1}-z_{0}}{Z \mu-1}\right)
$$


where $\mu=\frac{z_{2}-z_{0}}{z_{2}-z_{1}}$, then the pull-back of the first integral of $\widetilde{\mathcal{R}}$ by $\psi$ is the following meromorphic function :

$$
\left(\psi^{*} F\right)(X, Z)=\frac{\left(Z^{2}-2 Z+2\right)^{2}}{Z^{2}(Z-2)^{2}}
$$

Finally, the foliation $\psi^{*} \widetilde{\mathcal{R}}$ is locally defined by the 1 -form $d Z=0$. Likewise, the pull-back of the slopes $Z_{1}$ and $Z_{2}$ by $\psi$ defines a 2-web such that the leaves verify the following differential equation :

$$
(\star \star):\left(\frac{d Z}{d X}\right)^{2}+\frac{(t-1) Z^{4}+(-4 t+4) Z^{3}+(4 t-8) Z^{2}+8 Z-4}{4 X(X-1)(X-t)}=0
$$

In summary, the 4-web $\psi^{*} \mathcal{W}\left(\infty, Z_{0}, Z_{1}, Z_{2}\right)$ is locally equivalent to the web $\mathcal{W}(\infty, 0, \beta,-\beta)$, where $\beta$ is a solution of $(\star \star)$. As the 4 -web $\mathcal{W}(\infty, 0, \beta,-\beta)$ has a constant cross-ratios equal to -1 and all the 3 subweb are hexagonal, we can deduce that it is locally parallelizable.

Open Access This article is licensed under a Creative Commons Attribution 4.0 International License, which permits use, sharing, adaptation, distribution and reproduction in any medium or format, as long as you give appropriate credit to the original author(s) and the source, provide a link to the Creative Commons licence, and indicate if changes were made. The images or other third party material in this article are included in the article's Creative Commons licence, unless indicated otherwise in a credit line to the material. If material is not included in the article's Creative Commons licence and your intended use is not permitted by statutory regulation or exceeds the permitted use, you will need to obtain permission directly from the copyright holder. To view a copy of this licence, visit http://creativecommons.org/licenses/by/4.0/.

\section{References}

Atiyah, M.F.: Complex fibre bundles and ruled surfaces. Proc. Lond. Math. Soc. 3(5), 407-434 (1955)

Atiyah, M.F.: Vector bundles over an elliptic curve. Proc. Lond. Math. Soc. 3(7), 414-452 (1957)

Camacho, C., Neto, A.L.: Geometric theory of foliations. Birkhäuser, Boston (1985)

Friedman, R.: Algebraic surfaces and holomorphic vector bundles. Universitext. Springer, New York (1998) Hartshorne, R: Algebraic geometry. Graduate Texts in Mathematics, No. 52. Springer-Verlag, New YorkHeidelberg (1977)

Loray, F., Pérez, D.M.: Projective structures and projective bundles over compact Riemann surfaces. Astérisque 323, 223-252 (2009)

Maruyama, M.: On classification of ruled surfaces. Kinokuniya, Tokyo (1970)

Mukai, S.: An introduction to invariants and moduli, vol. 81. Cambridge University Press (2003)

Pirio, L.: Sur les tissus plans de rang maximal et le problème de Chern. C. R. Math. Acad. Sci. Paris 339(2), 131-136 (2004)

Ripoll, O.: Détermination du rang des tissus du plan et autres invariants géométriques. C. R. Math. Acad. Sci. Paris 341(4), 247-252 (2005)

Weil, A.: Variété de Picard et variétés jacobiennes. In: Séminaire Bourbaki, Vol. 2, pages Exp. No. 72, 219-226. Soc. Math. France, Paris, (1995)

Publisher's Note Springer Nature remains neutral with regard to jurisdictional claims in published maps and institutional affiliations. 